\title{
La « narration mêlée » dans les Mémoires sur Napoléon (1836-1837) de Stendhal
}

The "Mixed Narration" in Stendhal's Mémoires sur Napoléon (1836-1837)

\section{Hélène Spengler}

\section{OpenEdition}

\section{Journals}

Édition électronique

URL : http://journals.openedition.org/recherchestravaux/899

ISSN : 1969-6434

Éditeur

UGA Éditions/Université Grenoble Alpes

Édition imprimée

ISBN : 978-2-37747-006-8

ISSN : 0151-1874

\section{Référence électronique}

Hélène Spengler, «La «narration mêlée » dans les Mémoires sur Napoléon (1836-1837) de Stendhal », Recherches \& Travaux [En ligne], 90 | 2017, mis en ligne le 15 juin 2017, consulté le 08 septembre 2020. URL : http://journals.openedition.org/recherchestravaux/899

Ce document a été généré automatiquement le 8 septembre 2020

(c) Recherches \& Travaux 


\section{La « narration mêlée » dans les Mémoires sur Napoléon (1836-1837) de Stendhal}

The "Mixed Narration" in Stendhal's Mémoires sur Napoléon (1836-1837)

Hélène Spengler

Maxime : sur chaque incident se demander : faut-

il raconter ceci philosophiquement ou le raconter

narrativement selon le système de l'Arioste ${ }^{1}$ ?

1 Il est avéré que la lecture "réactive ", la plume à la main, des historiens anciens et classiques par Henri Beyle autodidacte, puis des mémorialistes et des historiographes modernes dont Stendhal fournit des comptes rendus critiques pour le public anglais, a préparé et nourri sa pratique historienne ${ }^{2}$. Séparés par vingt années d'intervalle et une Révolution, les deux projets historiographiques que Stendhal a consacrés au sauveur de la Révolution par la suite devenu son fossoyeur - Vie de Napoléon, rédigé entre mai 1817 et l'automne 1818; Mémoires sur Napoléon, travaillé pour l'essentiel entre novembre 1836 et avril 1837 - sont demeurés sans suite. Mais, malgré cet inachèvement, le premier comme le second projet (quoi qu'en ait dit Mérimée ${ }^{3}$ ) témoignent d'une conscience aiguisée des exigences méthodologiques de la "nouvelle historiographie » en quête d'exactitude et d'impartialité. Dans le sillage de l'Histoire d'Angleterre de David Hume, les esquisses de Stendhal explorent les enjeux narratifs du récit historique, exhaussés par les épigones de Walter Scott : MM. Thierry, de Barante et Mignet - pour ne citer que ces pilotis de la réflexion stendhalienne sur l'écriture de l'histoire ${ }^{4}$.

2 Véritable expérimentation historiographique, le projet inédit, dans tous les sens du terme, des Mémoires sur Napoléon de 1836-1837, constitue un objet d'étude éminemment intéressant, à notre sens, parce que complexe et informé par des enjeux idéologiques ${ }^{5}$, méthodologiques, épistémiques et littéraires proprement indissociables. 


\section{Un chantier ambitieux, mais inachevé, défiguré et par là peu lisible}

3 "J'écris cette histoire telle que j'aurais voulu la trouver écrite par un autre, au talent près ", écrit significativement Stendhal dans un premier projet de préface à ses Mémoires sur Napoléon daté de $1836^{6}$. De fait, le chantier des Mémoires sur Napoléon mérite de retenir notre attention pour ses ambitions, mêmes si partiellement réalisées, pour ses partis pris, novateurs, pour son " originalité ${ }^{7}$ » de conception et de pensée. Il faut $\mathrm{du}$ reste concéder certaines trouvailles et reconnaître quelques bonheurs d'écriture dans cette œuvre laissée pour compte :

Les Mémoires sont un texte auquel Stendhal attachait beaucoup d'importance ; alors qu'il avait abandonné son manuscrit de 1818 à Milan, il place celui des Mémoires dans ses caisses quand il repart pour l'Italie en 1839. Il n'y retouchera pas, mais certains chapitres, dont le fameux chapitre "Milan en 1796 ", ont été repris, presque inchangés, dans La Chartreuse de Parme qu'il met en chantier quelque mois après l'abandon définitif de l'ouvrage historique ${ }^{8}$.

Entre histoire et roman - l'historiographie n'étant d'ailleurs pas disjointe de la littérature dans cette première moitié $\mathrm{du} \mathrm{XIX}^{\mathrm{e}}$ siècle -, Stendhal s'attache, dans le récit, à concilier érudition et pittoresque, objectivité et vivacité, pour satisfaire aux exigences complémentaires de la raison et de l'imagination, en empruntant donc tout à la fois aux deux écoles historiographiques antagoniques des années 1830: à l'«école philosophique ", dont Guizot et Thierry sont alors les meilleurs représentants, et à l'école «narrative » et pittoresque, illustrée par de Barante - trois auteurs lus de près par Stendhal, qui en a livré des comptes rendus suggestifs dans la presse anglaise ${ }^{9}$. Toutefois, lorsque, dans le contexte déjà désenchanté des années 1836-1837, Stendhal se (res)-saisit de ce sujet au fort potentiel polémique - l'interminable Révolution ${ }^{10}-$, c'est bien avec l'espoir de se démarquer des multiples publications et productions contemporaines par un dispositif d'écriture pour le moins singulier - Stendhal optant pour une relation polyphonique et critique, à la croisée des genres des mémoires et de l'histoire, de l'individuel et du collectif, et portant un regard rétrospectif tantôt nostalgique tantôt distancié sur une histoire récente et le touchant de près ${ }^{11}$. D'où le titre hybride, envisagé le 13 février 1837, de Mémoires sur la vie de Napoléon.

5 Ainsi le texte, génériquement inclassable, des Mémoires sur Napoléon se signale-t-il par la transgression des codes et des contraintes, comme si l'éthopée de l'énergie à son maximum imposait l'invention d'une forme neuve, sui generis, et l'élaboration d'une poétique de l'histoire moderne. Notre auteur ne revendique-t-il pas crânement, dans un projet d'adresse à $\mathrm{M}$. le Libraire, "la fatuité de n'imiter personne ${ }^{12}$ " ? Le premier éditeur du texte - Romain Colomb, cousin et exécuteur testamentaire de BeyleStendhal - s'en montre d'ailleurs tout effaré : « cet ouvrage », note-t-il en marge de feuillets par lui arrachés au premier volume, «diffère entièrement de tout ce qu'on a publié jusqu'à présent ${ }^{13}$ ». Fait révélateur, Romain Colomb n'aura de cesse de «purger de tous ses hors-d'œuvre » cet ouvrage composite, taillant dans le vif du texte pour tâcher de redonner une forme à la non-forme - et rendant par là quasiment illisible le dispositif d'écriture original conçu par Stendhal ${ }^{14}$. 


\section{Conjuguer les voix, pour confronter points de vue et visions : la narration mêlée de lettres}

6 Revenons donc, pour la mieux caractériser, sur l'hybridité historiographique (ou, si l'on préfère l'impureté générique) qui tient au caractère «mêlék ${ }^{15}$ » de la narration dans les Mémoires sur Napoléon. Deux régimes de discours alternent: d'une part, ce que Stendhal présente comme le «récit raisonnable ${ }^{16}$ » des événements (autrement dit, le récit historique, tel qu'a pu le définir Benveniste ${ }^{17}$ ) et d'autre part, en contrepoint, des fragments de narration à la première personne (relevant de ce que Benvéniste caractérise comme discours $\left.{ }^{18}\right)$, constitués de documents d'archives ou de témoignages : rapports écrits dans la chaleur de l'action, harangues reconstituées pour les besoins de la propagande, lettres ou fragments dictés par l'Empereur à ses compagnons d'exil ; autant de morceaux d'éloquence destinés à captiver le lecteur mais qui participent aussi de la recherche de preuves, condition d'une histoire savante, la plus informée et la plus neutre possible - si tant est que l'impartialité soit possible en histoire ${ }^{19}$ :

C'est-à-dire qu'après avoir raconté les batailles d'une manière succincte, je transcrirai les longs récits dictés par Napoléon à Sainte-Hélène. Ainsi un quart à peu près des deux premiers volumes sera copié dans les œuvres de Napoléon ${ }^{20}$.

7 Si la place du témoignage enté sur l'événement, entaché de subjectivité, fait aujourd'hui débat parmi les historiographes ${ }^{21}$, n'oublions pas que son utilisation allait de soi dans l'historiographie antique et classique, dont notre auteur est pétri. Ainsi l'utilisation du témoignage direct, aussi subjectif soit-il, n'apparaît aucunement problématique à Stendhal, dans la mesure où il se voit introduit, complété et éventuellement modalisé, par un compte-rendu explicatif, dans une démarche comparatiste et critique :

Napoléon, tout occupé de son récit, [...] était plein de son sujet, et supposant, comme les gens passionnés, que tout le monde devait le comprendre à demi-mot, quelquefois il est obscur. Alors on a placé, avant l'admirable récit de Napoléon, les éclaircissements nécessaires. L'auteur les a trouvés dans ses souvenirs ${ }^{22}$.

8 Mérimée souligne avec justesse combien cette proximité, ici revendiquée par Stendhal, aux faits rapportés dans les Mémoires sur Napoléon est contestable, dans la lettre, déjà évoquée, du 12 février 1837. Précisons que si Henri-Marie Beyle, futur Stendhal, a en effet participé à la (deuxième) campagne d'Italie, en 1801, comme sous-lieutenant de cavalerie affecté au $6^{\mathrm{e}}$ dragon et aide de camp du général Michaud, il n'a en revanche pas été un témoin oculaire de la première campagne d'Italie de 1796-1797, étant alors élève à l'École centrale de l'Isère. Il reste que, nommé adjoint aux commissaires des guerres (en 1806), puis auditeur au Conseil d'État (en 1810) et Inspecteur de la Comptabilité du Mobilier et des Bâtiments de la Couronne, Beyle-Stendhal a participé à l'épopée impériale et a été un acteur et spectateur engagé de la campagne de Vienne (en 1809) puis de celle, désastreuse, de Russie (en 1812), enfin de celle de France (en 1814). Stendhal s'estime donc autorisé à construire son éthos comme ayant le «tour d'esprit " nécessaire à l'historien "pour voir les événements comme ils se sont vraiment passés ${ }^{23}$ ». Or, ce que l'on peut appeler, à la suite de Renaud Dulong, le pouvoir de «factualisation ${ }^{24}$ » de la mise en récit apparait bien comme essentiel à Stendhal: «[...] Substitu[er] la dissertation au récit. C'est le défaut de tous nos historiens" déplore-t-il dans une chronique pour la presse anglaise datée du 5 mars $1828^{25}$. Voyons ce qu'il écrit à propos de l'un d'eux, le 30 juin 1828 : 
[L'historien Robertson] que l'on continue d'admirer en France, a adopté dans son Histoire de Charles Quint l'idée singulière de rejeter dans les notes à la fin de son ouvrage tout ce qui était intéressant, tout ce qui était pittoresque, et tout ce qui était fait pour graver dans l'esprit un trait historique. [...] Or qu'est-ce que l'histoire si ce n'est l'art de représenter les événements comme ils se sont réellement passés ${ }^{26}$ ?

Autre commentaire significatif de Stendhal chroniqueur des historiographes contemporains, en mars 1825: "Il serait souhaitable que Mignet écrivît une autre histoire de la Révolution, avec plus de détails. En attendant, les meilleurs guides pour connaître cette époque extraordinaire sont [1]es sept cent quarante pages [de l'admirable ouvrage de M. Mignet sur la Révolution française] ainsi que quelques-uns des consciencieux mémoires relatifs à cet événement, comme les Mémoires de Thibaudeau, etc. ${ }^{27}$." Il apparait que Stendhal se lance dans son second projet sur Napoléon Bonaparte pour combler un manque - écrivant, "au talent près ", ce qu'il eût voulu lire sous la plume des historiographes, ses contemporains, comme le confirme une note autographe sur le manuscrit :

Le second tome relié doit commencer par: $1^{\circ}$ : récit raisonnable de la marche de flanc sur Plaisance. Passage du Pô, Fombio et Lodi. $2^{\circ}$ Récit des mêmes choses par Napoléon. [...] 10 février 1837. $1^{\circ}$ Dominique ne peut espérer que l'intérêt résultant de la narration. Son cœur lui dit ce qu'il y a à prendre dans un mauvais livre comme Bourrienne ou Rovigo. Il hait le ton dogmatique. Ce ton d'ailleurs serait déplacé dans un temps de partis et de méfiance. Donc, narrer, donc 5 ou 6 volumes. $2^{\circ} \mathrm{Ne}$ take le texte de Napoléon que pour les récits militaires. Donc refaire le récit de Leoben, le récit de la paix de Campo Formio [...], le récit de Venise ${ }^{28}$.

Dans le dispositif complexe des Mémoires sur Napoléon, les deux modalités de récit philosophique et narratif, loin d'être incompatibles, doivent ainsi se compléter, coconstruisant l'intelligibilité des faits passés rapportés. Le récit raisonnable s'attache à développer un point de vue distancié sur les événements, nourri de l'expérience de la vie militaire de Stendhal, étayé par ses lectures et ses recherches documentaires; les multiples relais de parole donnés au témoin oculaire et protagoniste des événements livrent quant à eux une vision: l'effet de réel s'ajoute donc au récit explicatif ${ }^{29}$. Dans le cas des Mémoires sur Napoléon, le témoignage subjectif intéresse précisément Stendhal pour sa charge émotive :

Le même homme qui a fait a raconté. Quel bonheur pour la curiosité des siècles à venir! [...] Comme mon but est de faire connaître Napoléon plus que les événements, je ne crois pas devoir priver le lecteur du récit qu'il a donné lui-même des opérations militaires qui suivent l'armistice de Cherasco ${ }^{30}$.

11 Contrepoint de la "troisième personne » et de la "première personne ", intrication des modes d'énonciation, concurrence de l'histoire et des mémoires: les Mémoires sur Napoléon ouvrent un espace pour la confrontation des voix et des points de vue, selon une démarche comparatiste chère à Stendhal : dans la onzième « Lettre de Paris par le petit neveu de Grimm ", datée du 11 octobre 1825, annonçant une publication attendue par le grand public, Stendhal chroniqueur précise qu'«il serait curieux de comparer l'histoire de la Corse du général [Sébastiani] avec l'admirable résumé de cette histoire rédigé par Napoléon dans sa jeunesse et publié en $1823^{31} »$. Loin de relever de la facilité, le parti pris de la coexistence systématique d'une énonciation coupée de la situation d'énonciation - dans le récit raisonnable des faits - et d'une énonciation ancrée dans la situation d'énonciation - dans les témoignages - n'est pourtant pas sans générer de multiples problèmes : historiographiques et méthodologiques, mais aussi rhétoriques 
(problème du choix et de l'ordonnancement des matières), cognitifs enfin et esthétiques (avec le risque d'illisibilité du dispositif, du fait de la quantité d'attention limitée $d u$ lecteur). D'inévitables recoupements - bien que l'auteur s'efforce d'« arrang[er] le sommaire [...], de façon à ce qu'il y eût le moins de répétitions qu'il était possible ${ }^{32} »-$ sont le revers du dispositif de juxtaposition des mises en récit. Stendhal en est fort conscient, qui éprouve le besoin d'une longue justification procataleptique de ce choix encore au cinquième chapitre des Mémoires:

Rien n'eût été plus facile que de profiter de ces récits, en les abrégeant [...]. Cette façon d'agir m'eût semblé un sacrilège. Selon moi une histoire de Napoléon qui ne placerait pas sous les yeux du lecteur les récits de la campagne d'Italie, tels que ce grand homme les a laissés, ne pourrait prétendre en aucune façon à faire connaître son caractère [...] Un autre motif m'a porté à transcrire les récits que Napoléon a donnés de ses principales batailles. J'ai considéré que le lecteur qui ne connaît Napoléon que par la plupart des histoires de ce grand homme, publiées jusqu'ici, est obligé d'admirer sur parole son talent pour la guerre.

Or, j'ai cru voir qu'il n'était pas impossible de raconter ses batailles et de les faire comprendre même au lecteur non militaire. Avant 1790, un récit de ce genre eût été impossible ; [...] J'ai longtemps hésité à transcrire le long récit du siège de Toulon : ce siège pouvait s'expliquer fort bien en six pages; mais :

$1^{\circ}$ Le lecteur peut passer sans inconvénient le récit de Napoléon.

$2^{\circ} \mathrm{Ce}$ récit de la première victoire de ce grand général me semble extrêmement curieux.

$3^{\circ}$ Quelques recherches que j'aie faites auprès des contemporains, je n'ai pu découvrir de mensonges dans le récit qu'on va lire. Ce ne fut qu'après Lodi que Napoléon songea à être autre chose qu'un général de la république ${ }^{33}$.

12 La conjugaison des voix et la confrontation des visions avait néanmoins l'avantage de favoriser l'émergence d'un point de vue critique distancié: celui du lecteur et de l'historiographe à venir. Comme le note avec modestie Stendhal, dès le 21 mars 1818, c'est « un devoir pour [lui] qui [a] connu ce grand homme d'en laisser un portrait à la postérité afin que les Hume futurs ne puissent mentir ${ }^{34} »$. Mais toute liberté est octroyée au «lecteur pressé » de passer certains passages - quand un lecteur plus exigeant prendra intérêt à la contextualisation et au commentaire critique des documents d'époque :

J'engage le lecteur pressé à passer les douze lettres qui suivent. Ce sont des rapports officiels adressés au Directoire par le général en chef de l'armée d'Italie. J'en avais fait un résumé qui, bien que présentant plusieurs vérités accessoires auxquelles Napoléon ne pouvait donner place dans ses lettres, était beaucoup plus court ${ }^{35}$.

13 En lecteur attentif, dès 1806, de l'Histoire d'Angleterre de David Hume ${ }^{36}$, Stendhal sait combien le dispositif d'écriture et le style constituent en eux-mêmes des outils heuristiques ${ }^{37}$. La confrontation des points de vue, dans les Mémoires sur Napoléon, permet à Stendhal, épigone en ceci de Hume historien, d'apporter un éclairage nuancé sur les faits et les représentations: "J'ai tâché de n'être pas dupe ${ }^{38}$ ", souligne le mémorialiste, dans le projet de préface de 1836. Chacune des grandes séquences des Mémoires sur Napoléon s'ouvre ainsi sur un "sommaire rapide ${ }^{39}$ " des événements, élaboré à partir de la compilation de textes-pilotis ${ }^{40}$, ensuite illustré par l'insertion de récits faisant «scènes ${ }^{41}$ ", par la reprise, entre autres sources documentaires, de larges extraits du Mémorial de Saint-Hélène ${ }^{42}$ (source réputée plus fiable que les Mémoires pour servir à l'Histoire de la France, sous Napoléon, par Gourgaud et Montholon ${ }^{43}$ ) ou encore de la copie de pièces officielles tirées de l'Histoire de Napoléon ${ }^{44}$. 
14 De fait, le traitement des documents issus de la propagande impériale était chose délicate : «En sa qualité de souverain, Napoléon écrivant mentait toujours ${ }^{45}$. » Aussi Stendhal procède-t-il, comme en 1817-1818 pour The Life, au recoupement des informations, selon une technique d'assemblage exemplaire, particulièrement visible au chapitre 40, empruntant non seulement au Mémorial de Sainte Hélène, mais aussi à la Biographie des hommes vivants de Michaud ${ }^{46}$, et à différents Mémoires de contemporains mentionnés en marge des manuscrits, comme MM. Lavalette, Fauche-Borel et Bourrienne $^{47}$. L'insertion d'autres voix dans le « récit raisonnable» des événements ou à l'occasion d'anecdotes - comme l'histoire du lieutenant Robert, au chapitre 19, ou le récit des souvenirs du peintre Boguet rattachés au commentaire de la bataille de Rivoli, aux chapitres 37 à 39 des Mémoires sur Napoléon - était censée vérifier par les «plus petits détails » les déclarations propagandistes du héros de la campagne d'Italie comme celles des mémorialistes concurrents :

Tout le monde avoue que l'homme qui raconte doit dire la vérité clairement. Mais pour cela il faut avoir le courage de descendre aux plus petits détails. C'est là, ce me semble, le moyen unique de répondre à la défiance du lecteur. Loin de redouter cette défiance, je la désire et la sollicite de tout mon cœur ${ }^{48}$.

Dire la vérité clairement, pour émouvoir et convaincre : on retrouve ici une vertu de l'historiographie antique, qui donne à voir pour faire penser. Pesant le pour et le contre du choix d'une narration mêlée, Stendhal affirme en effet écrire « pour des gens qui ont lu Montesquieu ${ }^{49} \gg$ et sachant penser par eux-mêmes :

Comptant sur l'intelligence du lecteur, je ne garde point toutes les avenues contre la critique; les hypocrites m'accuseront probablement de manquer de morale, ce qui n'augmentera point la dose de mépris que j'ai pour ces gens-làs ${ }^{50}$.

Aussi, Stendhal, comme le fait observer le journaliste et critique Auguste Bussière, son contemporain :

[...] émet peu de propositions qu'il ne fasse suivre de cet avis renouvelé sous toutes les formes : "J'invite à se méfier de tout le monde, même de moi... Ne croyez jamais qu'à ce que vous avez vu, n'admirez que ce qui vous fait plaisir, et supposez que le voisin qui vous parle est un homme payé pour vous mentir"51.

17 Ce sont là, ajoute Auguste Bussière, autant de " précautions qu'il prend pour forcer son lecteur à penser ou à le prendre en haine ${ }^{52} »$. Car il s'agit pour Stendhal de former le jugement et de «conduire les idées du lecteur ${ }^{53}$ ». L'on sait que les relecteurs de la première Vie de Napoléon de 1817-1818 étaient invités à s'approprier l'ouvrage en crayonnant commentaires et critiques dans les marges ${ }^{54}$. Invitant le lecteur potentiel des Mémoires sur Napoléon à se faire une idée par lui-même, et non "sur parole ", du caractère singulier et du génie militaire de Bonaparte, Stendhal le renvoie explicitement aux documents consultables à son époque et - ce qui doit retenir notre attention - aux cartes géostratégiques alors disponibles.

\section{L'invention d'une méthode historienne et esthétique}

Le renvoi systématique aux cartes, désormais accessibles au large public, témoigne d'une véritable exigence méthodologique, qui passe par l'intertextualité. Pour autant, l'enjeu n'est pas exclusivement méthodologique, mais aussi rhétorique et esthétique, comme nous allons le voir dans le cas des batailles de la vallée de l'Adige, où le renvoi aux cartes existantes est particulièrement insistant. « Nous allons entrer dans le récit d'opérations admirables; mais pour qu'il puisse être sensible à ce qu'elles ont de 
sublime, je supplierai le lecteur de regarder une fois une carte passable du lac de Garde ${ }^{55}$ » souligne Stendhal au chapitre 23 des Mémoires sur Napoléon, en guise de prélude à sa propre description du cadre (bucolique) des grandes manœuvres militaires de Castiglione, d'Arcole et de Rivoli :

Si le lecteur veut se rendre compte de la singularité de ce plan ["vraiment allemand » d'Alvinzi de subdiviser ses forces], il peut vérifier sur une bonne carte géographique que, par une suite d'obstacles naturels et invincibles, aucune de ces colonnes ne pouvait communiquer avec sa voisine.[...] Depuis l'offensive de Provera, le $1^{\text {er }}$ janvier 1797 , jusqu'à la reddition de Mantoue, le $1^{\text {er }}$ février suivant, espace d'un mois (Voy[ez] la carte) ${ }^{56}$.

19 Pour «donner au lecteur la sensation d'assister à toutes les scènes qu'il décrit et de participer à toutes les actions qu'il raconte ${ }^{57}$ ", Stendhal esquisse, plus qu'il ne dessine, les renvois à des documents extratextuels : tableaux, gravures, cartes se substituent au récit épique et à la rhétorique de l'hypotypose attendus. Examinons les diverses représentations concurrentes de l'inénarrable bataille de Rivoli qui plaça Bonaparte «au premier rang des plus grands capitaines». Pour rendre compte de l'une des victoires les plus fameuses du général - la bataille de Rivoli ayant été popularisée par d'innombrables gravures et comptes rendus ${ }^{58}-$, Stendhal, à l'instar du peintre Boguet expressément chargé par Bonaparte d'en fixer le souvenir, et différant sans cesse la livraison obligée du "tableau ${ }^{59}$ ", multiplie les esquisses et les prises de vue préparatoires. Tel qu'il nous est conservé, et tout mutilé qu'il soit par les interventions de Colomb ${ }^{60}$, le manuscrit des Mémoires témoigne des recherches de l'auteur tâtonnant entre une esthétique du "trop fini ", laquelle a l'inconvénient de figer l'imagination par une trop grande intervention de la raison (grand défaut du néo-académisme selon Stendhal) et une esthétique du «trop esquissé61 ${ }^{\prime}$. Mais aussi, comment restituer par des mots pareil déploiement d'énergie et de forces en mouvement?

Paraphrasant Jomini ${ }^{62}$, le chapitre 37 constitue le sommaire raisonné et raisonnable des événements : «Ici finit rérais ${ }^{63}$ » lit-on à l'explicit du chapitre, à la date du 25 mars 1837. Le morceau de bravoure attendu des lecteurs, le «tableau » sublime de la bataille, en hypotypose, n'est pas donné ou, du moins, seulement en creux, au chapitre 38: de ce tableau qui peindrait en acte et ferait voir la bataille, nous n'avons que l'esquisse, à travers l'évocation du récit qu'improvisèrent le général Berthier et Napoléon Bonaparte en personne, peu après les événements, devant le peintre Boguet ${ }^{64}$. Le général en chef de l'armée d'Italie pressait ce dernier de fixer sur la toile le souvenir de sa récente victoire sur le plateau de Rivoli :

Le général Berthier dessina les divers mouvements de la bataille : le Monte-Baldo à gauche, la hauteur San Marco en face, l'Adige vers la droite. / Et ce fut sur cette sorte de plan improvisé que Napoléon, fort en train de causer et de discuter, et Berthier, tâchèrent de faire comprendre au peintre les mouvements successifs que nous venons de raconter. Le peintre était électrisé par un si beau récit fait, disait-il, avec la dernière simplicité et sans la moindre emphase. [...] Sans doute Napoléon espérait avoir un tableau de bataille. Autrement, dit M. Biogi [Boguet], à quoi bon expliquer avec tant de netteté les mouvements des troupes et surtout les différences de leurs uniformes. Les canonniers, avec leurs pièces de douze, plongeant à droite dans la vallée de l'Adige et labourant les troupes de Quasdanowitch en uniforme blanc, qui veulent monter sur le plateau; les dragons, en habits verts, commandés par Lassalle, etc., etc. / On se sépara à plus de deux heures après minuit ${ }^{65}$.

21 Le chapitre 39 présente enfin "le récit de Napoléon ", copié du Mémorial de SainteHélène. De la criante insuffisance de la parole à peindre, partiellement comblée par 
l'insertion d'images extratextuelles, témoignent ces deux notes additionnelles de Stendhal à la copie du Mémorial : "voyez la carte "; "voir le magnifique paysage de M. Boguet au Luxembourg ${ }^{66} »$. Mais est-ce là un aveu d'impuissance ou une trouvaille esthétique? Il nous semble que, par un très intéressant effet de mise en abyme, les explications fournies par Napoléon au peintre Boguet pour pouvoir (se) représenter la bataille de Rivoli constituent une invitation expresse au lecteur à choisir les couleurs et remplir lui-même les blancs de la fresque, par la force de son imagination. Renonçant à toute reconstitution, comme à l'ekphrasis du tableau effectivement peint par Boguet, Stendhal choisit d'épurer son propos, laissant à l'auditeur-lecteur le soin d'imaginer lui-même ce qui n'est pas représenté, mais esquissé. Or, parce que l'imagination, « rejetant l'objet sous les sens, est flattée par la promesse de quelque chose de plus », la simple esquisse procure au spectateur, ainsi que l'avait constaté Burke, une "jouissance " esthétique "bien plus vive que le dessin le mieux fini ${ }^{67}$ ». Aussi l'évocation en pointillés de la scène de bataille, au chapitre 38 , se révèle-t-elle particulièrement suggestive, selon l'esthétique stendhalienne de l'ellipse (« etc., etc.») et de la retenue, sans la moindre emphase. Voyez l'adresse de Stendhal «à Monsieur le Libraire » :

\begin{abstract}
Je vous demande pardon, Monsieur, il n'y a nulle emphase dans les volumes que l'on vous présente à acheter. S'ils étaient écrits en style Salvandy, on vous demanderait quatre mille francs par volume. Il n'y a jamais de grandes phrases ; jamais le style ne brûle le papier, jamais de cadavres; les mots horrible, sublime, horreur, exécrable, dissolution de la société, etc., ne sont pas employés. [...] J'ai cherché à raconter non pas comme MM. De Salvandy ou de Marchangy, mais comme Michel de Montaigne ou le président de Brosses ${ }^{68}$.
\end{abstract}

Les avantages et inconvénients de cette esthétique de l'esquisse et du non-finito étaient pesés depuis longtemps par Stendhal ${ }^{69}$. Comme le souligne Giuseppe Tomasi di Lampedusa, "dans l'Histoire de la peinture en Italie, Stendhal a déjà trouvé sa propre méthode esthétique ", savoir "mettre en jeu l'imagination du spectateur " par une «négligence apparente qui fait voir au spectateur ce qu'on ne peut pas lui montrer ${ }^{70}$ ».

\title{
Un pacte de lecture exigeant
}

Intelligenti pauca. Le pacte de lecture, dans les Mémoires sur Napoléon, est pour le moins exigeant, le lecteur étant appelé à participer, par sa mémoire propre (pour les témoins des faits), par son intellect et par son imaginaire, à la constitution d'un savoir intrinsèquement tissé de " croyances ", comme l'avait établi Hume, mais passant par la déconstruction des représentations et croyances communes ${ }^{71}$.

Un texte antérieur de Stendhal : Rome, Naples et Florence en 1817 avait explicitement mis en scène (et en abyme) ce processus dialectique entre gens d'esprit réunis, par-delà les clivages idéologiques, au-dessus d'une carte de bataille, le croquis de la défaite de Tolentino, levé à la boussole par le personnage-narrateur (un certain Monsieur de Stendhal, officier de cavalerie allemand) fournissant l'occasion d'un riche échange de points de vue avec un colonel anglais ${ }^{72}$.

Quelle préfiguration, en outre, dans cet avant-texte, des actuelles possibilités de l'hypertexte! Appréhendons donc le chantier des Mémoires sur Napoléon moins comme une «œuvre-fatras ${ }^{73}$ », selon le trop sévère jugement de Giuseppe Tomasi di Lampedusa, que comme le projet stimulant d'une « œuvre ouverte ${ }^{74} »$. 


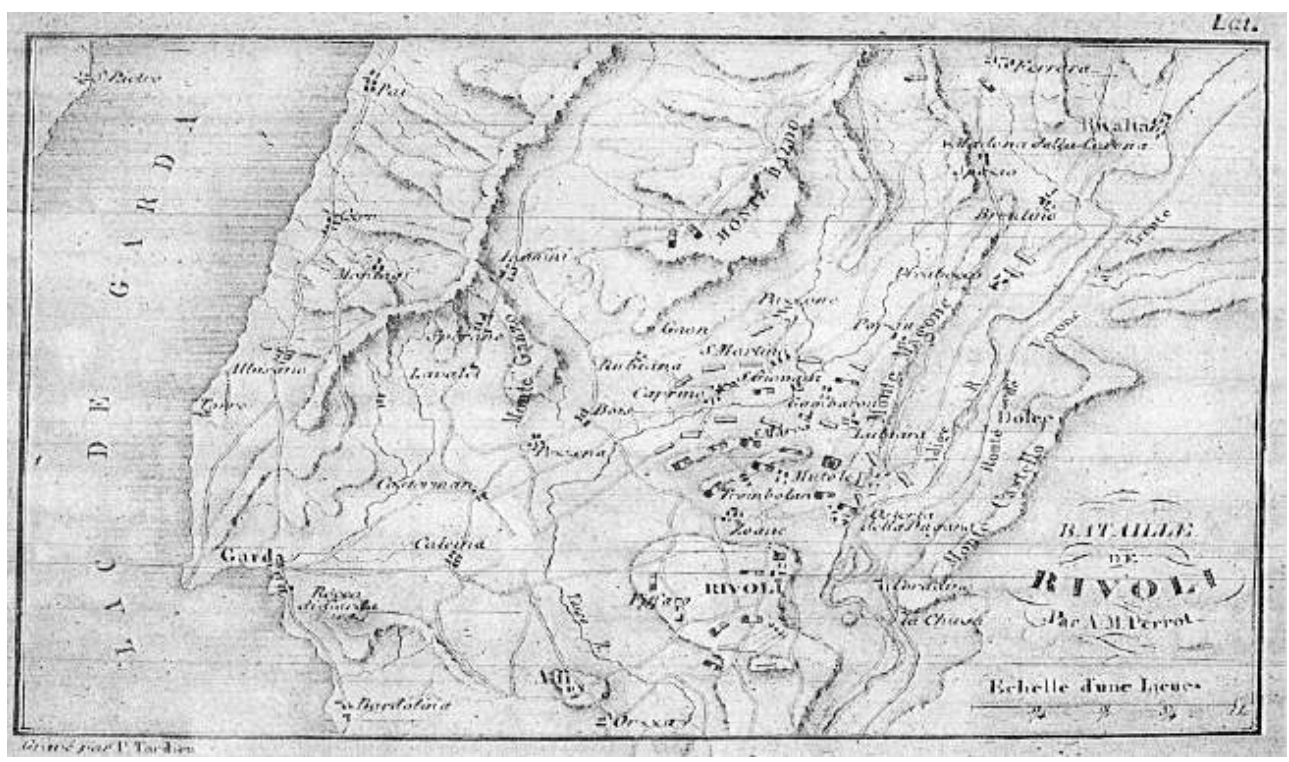

Bataille de Rivoli, 25-26 Nivôse an V [14-15 janvier 1797], par A. M. Perrot (Paris, BNF, Histoire de France, [M103575]). Tous droits réservés.

\section{NOTES}

1. Stendhal, note du 2 octobre 1839 (Euvres intimes, Paris, Gallimard, 1982, coll. « Bibliothèque de la Pléiade ", t. II, p. 354).

2. Nous reprenons l'expression d'écriture "réactive " à Gérald Rannaud. Voir C. Meynard, «La genèse d'une écriture de l'histoire chez Stendhal. Ecriture-mosaïque et intertextualité dans Histoire d'Espagne (1808) », Genesis, n² 29, 2008, p. 65-77 ; V. del Litto, La Vie intellectuelle de Stendhal. Genèse et évolution de ses idées, Paris, PUF, 1959, p. 187- 211, p. 294-306, p. 346-377. De Stendhal, on pourra consulter les Journaux et papiers. Volume I - 1797-1804, édité par C. Meynard, H. de Jacquelot et M.-R. Corredor, Grenoble, Ellug, 2013, vol.1, p. 357, p. 565 notamment. Les comptes rendus critiques des ouvrages de Barante, de Thierry, de Guizot, ou élogieux de Ségur par Stendhal, ont été recueillis dans les Chroniques pour l'Angleterre, Grenoble, Ellug, 1980 et sont repris dans ParisLondres, Paris, Stock, 1997 : « L'histoire est ce que nous savons le mieux faire en France ; pourtant, tous les livres d'histoire qui ont été écrits depuis dix ans doivent quelque chose à sir Walter Scott » écrit Stendhal le 22 mai 1828 (p. 863); aussi «tous nos vieux historiens pâlissent devant MM. Thierry, de Barante et Mignet» (p. 543).

3. Dans une lettre critique, envoyée à Stendhal en date du 12 février 1837, Mérimée disqualifie la préface - sinon le projet lui-même - des Mémoires sur Napoléon pour son « manque complet de méthode ». Voir à ce sujet l'« Introduction » de C. Mariette à son édition Napoléon. Vie de Napoléon. Mémoires sur Napoléon [désormais abrégé MN], Paris, Stock, 1998, p. X.

4. Voir S.-A. Leterrier, Le XIX siècle historien. Anthologie raisonnée, Paris, Belin, 1999.

5. La parole de l'historien est toujours située: « on vous prendra pour un républicain, malgré votre protestation de la dernière page en faveur de l'état de choses actuel » met ainsi en garde Mérimée dans sa lettre du 12 février 1837 (MN, p. 699.) 
6. Voir le « Premier projet de préface (1836)» (MN, p. 249).

7. «Pourquoi ai-je conduit ainsi les idées du lecteur ? (13 février 1837). Préface pour moi » (MN, p. 247).

8. Introduction de C. Mariette à Napoléon, ouvr. cité, p. XVIII.

9. Sur les deux exigences d'exactitude et de pittoresque nécessaires à l'histoire, voir les commentaires de Stendhal pour ses lecteurs anglais dans Paris-Londres, ouvr. cité, notamment p. 702-703 : Stendhal y confronte Guizot et Hume. Concernant Guizot, voir aussi, p. 155, 476, 510, $574,619,833$ et 874 ; concernant Thierry, voir, p. 410, 428, 574; concernant de Barante, voir p. 198-200, 263, 276, 427-428, 476, 646, 833; concernant Mignet, voir p. 173 et 248 ; concernant Thiers, voir p. 248, 338, 625-627.

10. Comme le rappelle plaisamment R. Debray, «La Monarchie de Juillet a tellement répété "la Révolution est terminée" qu'elle en a d'ailleurs rendu inévitable une deuxième, 1848. " (Que vive la République, Paris, Odile Jacob, 1989, ch. 3 : «La Révolution française est interminable », p. 54). Sur les courants et conditions politiques sous la Monarchie de Juillet, voir D. Barjot, J.-P. Chaline, A. Encrevé, La France au XIXe siècle, 1814-1914, Paris, PUF, 1995, p. 169-212.

11. Voir C. Mariette, «La notion de "récit raisonnable" dans les Mémoires sur Napoléon », L'Année Stendhal, $\mathrm{n}^{\circ} 2$, Paris, Klincksieck, 1998, p. 51-61 et C. Mariette, «La vérité en crise dans les Mémoires sur Napoléon de Stendhal ", Le Sens du passé, pour une nouvelle approche théorique des Mémoires, de Commynes à nos jours, Marc Hersant, Jean-Louis Jeannelle, Damien Zanone (éd.), La Licorne, Presses universitaire de Rennes, 2013.

12. $M N$, p. 245.

13. MN, p. 705 (note a).

14. Les traces du découpage effectué par le premier éditeur du texte, R. Colomb, sont fort nettement visibles dans le troisième volume du manuscrit relié de la «Vie de César» (Bibliothèques municipales de Grenoble, ms R 288 (3), fo 88 à 170) - épuration qui ne fut pas sans conséquence sur l'interprétation critique de l'œuvre. Quant à l'Histoire des éditions successives, on se reportera à l' "Introduction" de C. Mariette à Napoléon, ouvr. cité, p. XV. Voir aussi C. Mariette, "Le dossier manuscrit des Mémoires sur Napoléon", dans Stendhal à Cosmopolis, Stendhal et ses langues, M.-R. Corredor (éd.), Grenoble, Ellug, 2007, p. 339-342.

15. Dans une note datée du 6 août 1804, H. Beyle se proposait de combiner la forme épistolaire au récit pour composer un roman, par une "narration mêlée de lettres" (Pensées, Filosofia Nova, Paris, Le Divan, 1931, t. II, p. 239). Nous reprenons la formule stendhalienne en lui donnant une plus grande extension.

16. Voir MN, p. 247 et 249.

17. Voir É. Benvéniste, Problèmes de linguistique générale, Paris, Gallimard, 1966, t. I, ch.19, p. 237-250 (p. 239-241).

18. Ibid., p. 241-242 : «Il faut entendre discours dans sa plus large extension : toute énonciation supposant un locuteur et un auditeur, et chez le premier l'intention d'influencer l'autre en quelque manière. "

19. Voir à ce sujet V. Ferry, Traité de rhétorique à usage des historiens, Paris, Classiques Garnier, 2015, p. 210-212: « l'illusion de la neutralité».

20. Voir MN, p. 288. Notons que la collection (dirigée par F. Guizot) des Mémoires relatifs à l'histoire de France, depuis la fondation de la monarchie française, jusqu'au XIII 'siècle (Paris, chez J.-L.-J. Brière, 1823), prévoit et encourage la reproduction de "curieux documents » : voir à ce sujet le compte rendu par Stendhal de la douzième livraison, dans Paris-Londres, ouvr. cité, p. 619.

21. Voir V. Ferry, Traité de rhétorique à usage des historiens, ouvr. cité, p. 80-82: «le témoignage comme preuve en histoire »; H. Wallenborn, L'Historien, la parole des gens et l'écriture de l'histoire : le témoignage à l'aube du XXI siècle, Bruxelles, Labor, 2006.

22. MN, p. 249 : premier projet de préface (1836). Nous soulignons.

23. Voir Paris-Londres, ouvr. cité, p. 873 , nous soulignons. 
24. Voir R. Dulong, Le Témoin oculaire. Les conditions sociales de l'attestation personnelle, Paris, Éditions de l'EHESS, 1998, p. 211.

25. Voir Paris-Londres, ouvr. cité, p. 833.

26. Ibid., p. 873 (Voir Robertson, Histoire du règne de l'empereur Charles-Quint, Paris, Chez Saillant \& Nyon 1771, 6 tomes en 6 vol. in-12 $2^{\circ}$.

27. Voir Paris-Londres, ouvr. cité, p. 338 (nous soulignons). Voir F.-A. Mignet, Histoire de la Révolution française depuis 1789 jusqu'en 1814, Paris, Firmin Didot père et fils, 1824 ; A. C. Thibaudeau, Mémoires sur la Convention et le Directoire, Paris, Baudouin frères libraires, 1824.

28. Voir MN, p.X (Bibliothèques municipales de Grenoble, ms R 288, I, fo 447 verso, note autographe de Stendhal).

29. Voir C. Ginzburg, "Montrer et citer. La vérité de l'histoire ", dans Le Débat, $\mathrm{n}^{\circ} 56,1989$, p. 41-51.

30. $M N$, p. 249 et 381 . Napoléon en effet, « est de ces hommes qu'on ne peut abréger, parce que leurs paroles peignent leurs sentiments» (MN, p. 249 : premier projet de préface). Et « qui oserait après Napoléon faire le récit de la bataille d'Arcole ?» (MN, p. 514).

31. Paris-Londres, ouvr. cité, p. 574.

32. $M N$, p. 381.

33. $M N$, p. 287-288.

34. Voir Euvres intimes, ouvr. cité, t. II, p. 5 (nous soulignons). Stendhal travaillait alors à prolonger la première Vie de Napoléon par L'Italie en 1818, dont les fragments sont recueillis dans Voyages en Italie, Paris, Gallimard, 1973, coll. « Bibliothèque de la Pléiade », p. 179-284.

35. MN, p. 514-515. Nous soulignons.

36. Voir Euvres intimes, ouvr. cité, t. I, p. 436-438; p. 709 et 710 : Stendhal note dans ses journaux avoir lu « avec la plus grande attention » en mai 1806 « the excellent essay of Hume»; p. 934 . Dans ses chroniques pour l'Angleterre, il lui reproche néanmoins de ne pas savoir « peindre » assez en détail l'histoire (voir Paris-Londres, ouvr. cité, p. 872).

37. Voir C. Gautier Hume et les savoirs de l'histoire, Paris, Librairie philosophique Vrin/ Éditions de l'EHESS, coll. «Contextes », 2005, Première partie, ch. III : «La balance comme dispositif de neutralisation », p. 136-145.

38. $M N$, p. 249.

39. $M N$, p. 403.

40. Sur les principales sources bibliographiques utilisées par Stendhal voir l'appendice de C. Mariette à son édition, Napoléon, ouvr. cité, p. XIX-XX.

41. Au sens où l'entend G. Genette dans son « Discours du récit » (voir Figures III, Paris, Éditions du Seuil, 1972, coll. « Poétique », p. 141-144).

42. Voir le Mémorial de Sainte-Hélène ou Journal où se trouve consigné, jour par jour, ce qu'a dit et fait Napoléon durant dix-huit mois, par le comte de Las Cases, Paris, 1823, 8 vol. in-8 ${ }^{\circ}$.

43. Les Mémoires pour servir à l'Histoire de France, sous Napoléon, écrits à Sainte Hélène par les généraux qui ont partagé sa captivité, et publiés sur les manuscrits entièrement corrigés de la main de Napoléon, par les barons Gourgaud et Montholon, avaient paru chez Firmin Didot-père et fils, à Paris, entre 1823 et 1825,6 vol. in- $8^{\circ}$.

44. Il s'agit de l'Histoire de Napoléon d'après les mémoires écrits à Sainte-Hélène sous la dictée de ce prince, par les généraux Montholon et Gourgaud, le comte Las Cases, le docteur O'Meara, et les historiens les plus impartiaux du siècle, publiée par J. Dourille, Paris, Constant-Chantpré, 1829, 2 vol. in-8.

45. Voir MN, p. 249 ; voir aussi $M N$, p. 462 : «On pense bien que sa correspondance avec ce gouvernement inhabile et malveillant, n'était pas un modèle de franchise ; toutefois, afin de donner quelque idée de ces temps déjà si loin de nous et d'ailleurs, il faut le dire, par admiration pour ces lettres, nous en placerons ici quelques-unes. / Malgré leur longueur, on en avait fait un résumé, mais tout ce qui peint ces temps de désordre et de grandeur disparaissait et prenait une fausse apparence. » 
46. L. G. Michaud a coordonné une Biographie des hommes vivants ou histoire par ordre alphabétique de la vie publique de tous les hommes qui se sont fait remarquer par leurs actions ou leurs écrits, ouvrage entièrement neuf rédigé par une Société de gens de lettres et de savants, Paris, 1816-1819, 5 vol. in-8².

47. Voir MN, p. 732, note * : «Le public n'a accordé aucune confiance aux publications de M. Fauche-Borel et Bourrienne, de ce dernier surtout. Quand je cite un fait publié par ces écrivains, c'est que je l'ai trouvé dans des Mémoires manuscrits ou qu'il m'a été confirmé par des personnes qui se trouvaient auprès du général Bonaparte de 1797 à 1802.11 février 1837. »

48. $M N$, p. 251 : Deuxième projet de préface (1837).

49. $M N$, p. 250 : Premier projet de préface (1836).

50. Ibid.

51. Voir A. Bussière, "Henri Beyle (M. de Stendhal) », article repris dans Stendhal, mémoire de la critique, Paris, Presses de l'université de Paris-Sorbonne, 1996, p. 173.

52. Ibid., p. 175 et 180.

53. Voir MN, p. 247.

54. Rappelons que le manuscrit relié du premier projet: la Vie de Napoléon de 1817-1818 avait circulé sous le manteau et porte dans ses marges et sur les derniers feuillets réservés les commentaires de deux relecteurs : Vismara et Borsieri.

55. $M N$, p. 418.

56. $M N$, p. 543 et 553 (Bibliothèques municipales de Grenoble, ms R 288 (3), fo 171 ).

57. Voir l'éloge par Stendhal, journaliste pour la presse anglaise, de son propre ouvrage De l'Amour (Paris-Londres, ouvr. cité, p. 93).

58. D'après A. Dayot (voir Napoléon raconté par l'image, Paris, Librairie Hachette et $C^{\text {ie }}, 1902$ ), Rivoli est, après le pont d'Arcole, la scène la plus représentée de la geste napoléonienne. Le Cabinet des Estampes en conserve plusieurs versions gravées et diffusées dans la presse populaire (Bibliothèque nationale de France, Histoire de France ; Qb1 1797).

59. Voir MN, p. 547-549.

60. Le carton coté $\mathrm{R} 288$ bis renferme les brouillons autographes de deux chapitres correspondant aux feuillets manquants dans le troisième volume relié de la "Vie de César " (Bibliothèques municipales de Grenoble, ms R 288 (3), fo 88 à 170), qui furent découpés par R. Colomb dans le projet d'une édition des Euvres complètes de Stendhal. Il s'agit des chapitres 37 ( $f^{\circ} 108$ à 153 ) et 38 (fo 155 à 156), tels que reconstitués dans l'édition de C. Mariette (Bibliothèques municipales de Grenoble, ms R 288 bis, respectivement fo 9 à 22 et fo 23 à 29 , dans la nouvelle cotation des bibliothécaires).

61. M. Crouzet, "Stendhal et la poétique du fragment", Stendhal Club, nº 94, 15 janvier 1982, p. 157-180 (p. 160). Concernant le pouvoir d'emportement du narratif chez Stendhal, et les deux modes de récit philosophique et narratif, voir M. Crouzet, Le Naturel, la Grâce et le Réel dans la poétique de Stendhal, ouvr. cité, $\mathrm{IV}^{\mathrm{e}}$ partie ch. 21 : «Le réel et le récit», p. 300-314. Voir aussi W. A. Guentner, « Rhétorique et énergie », Romantisme, 1984, n 46, p. 27-35 (p. 34).

62. Voir Jomini, Vie politique et militaire de Napoléon racontée par lui-même au tribunal de César, d'Alexandre et de Frédéric (Paris, 1827, 4 vol in-8º, p. 185-191).

63. « Rérais » [c'est-à-dire le « récit raisonnable » des événements]. Note autographe au verso du fo 153 , recoté fo 22 verso (MN, p. 730 , note d.)

64. Pages arrachées au troisième volume du manuscrit relié (correspondant aux fo155 à 166) et dont des fragments de brouillons nous ont été conservés (Bibliothèques municipales de Grenoble, ms carton R 288 bis, fo $23-29$, dans la nouvelle cotation bibliothèque).

65. $M N$, p. 549-550.

66. MN, p. 553 et 558, note*. Le tableau de Boguet en question est aujourd'hui conservé au Musée de Périgueux.

67. Voir E. Burke, Recherche philosophique sur l'origine de nos idées du sublime et du beau [1757], trad. française de Lagentie de Lavaisse, Partie II, section XI : «L'infinité dans les objets agréables »: 
«Souvent j'ai trouvé dans une simple esquisse un je-ne-sais-quoi qui me procurait une jouissance bien plus vive que le dessin le mieux fini. » (Paris, Vrin, 1973, p. 139.)

68. $M N$, p. 245. À rapprocher d'un fragment daté de Milan, le 12 août 1814 : «Le style affecté, qui fait passer les idées communes, nuit à l'effet des idées vraiment grandes. Il est obligé de les charger d'épithètes, tandis que dans le style simple une seule épithète mise pour peindre une idée vraiment grande produit tout l'effet désirable. Ce style simple doit ennuyer les sots et plaire beaucoup plus aux âmes grandes. » (Mélanges de Littérature, Paris, Le Divan, 1933, t. III, p. 127).

69. Voir Histoire de la Peinture en Italie, Paris, Gallimard, 1996, p. 418 : dans le genre terrible, note Stendhal, le non finito est " presque une grâce ».

70. Voir G. Tomasi di Lampedusa dans son essai sur Stendhal, traduit de l'italien par Monica Baccelli, Paris, Éditions Allia, 2002, p. 33.

71. Voir C. Gautier, Hume et les savoirs de l'histoire, ouvr. cité, Première partie : "L'Histoire comme pratique. Discrimination entre les croyances politiques communes ».

72. Voir Voyages en Italie, ouvr. cité, p. 101-102 : «Avant-hier, comme je levais à la boussole un croquis de la bataille de Tolentino [humiliante défaite des troupes de Murat, roi de Naples depuis 1808, face aux Autrichiens dans les Marches, le 2 mai 1815], je remarquai une figure militaire, aussi à cheval, qui suivait mes mouvements. Nous nous trouvâmes, le soir, à l'auberge de Macerata ; et l'ennui, ce grand mobile des gens d'esprit fit que le colonel Forsyt m'adressa la parole. Voyant un homme âgé, je lui offris une copie de mon plan : il accepta. [...] Accoutumé à ce travail dans les états-majors, j'eus bientôt dépêché ma petite carte. Sensible à cette marque d'attention, mon colonel, qui m'avait suivi dans ma chambre, voulut être aimable pour moi et parla presque autant qu'un Français. [...] Sûrs de nous quitter demain, probablement pour toujours, nous nous hâtons, mon colonel et moi, de nous dire, en peu de mots, tout ce que nous avons de plus intéressant. "

73. C'est là l'injuste reproche de G. Tomasi di Lampedusa à l'encontre des deux versions de la Vie de Napoléon de 1818 et 1836 (voir Stendhal, ouvr. cité, p. 37 et 46).

74. Pour reprendre le titre de l'essai d'U. Eco (voir L'Euvre ouverte, traduit de l'italien par C. Roux de Bézieux et A. Boucourechliev, Paris, Le Seuil, 1965).

\section{RÉSUMÉS}

Dans ses Mémoires sur Napoléon (1836-1837), Stendhal élabore un dispositif complexe, à travers une narration mêlée d'extraits choisis, de documents et de témoignages contemporains ou immédiatement postérieurs aux faits, destinés à permettre aux lecteurs de la Monarchie de Juillet comme aux historiographes futurs de se construire leur propre vision du général Bonaparte, futur Napoléon $\mathrm{I}^{\mathrm{er}}$. La sélection et la mise en perspective de documents historiques et de témoignages, le recours et le renvoi systématique aux cartes géostratégiques disponibles, témoignent du travail de recherche par Stendhal d'une écriture moderne de l'histoire, issue de David Hume et Walter Scott, mais selon un dispositif inédit et intrinsèquement problématique. Inabouti, défiguré par les interventions du premier éditeur du manuscrit, le projet stendhalien manque sans doute de lisibilité, mais non d'intérêt, dans sa double ambition méthodologique et esthétique.

In his Memoirs On Napoleon (1836-1837), Stendhal develops a complex double narration with a choice of contemporary documents and accounts, so that both readers in the present and 
historians in the future could make up their own mind about Napoleon Bonaparte. The set of historical traces and the use of geostrategical maps give proof of real strictness of Stendhal and his manuscript shows the methodological and literary research of a modern way, for a new historiography and a powerful style. We also shall comprehend the Memoirs On Napoleon in his historical and aesthetic dimensions, as a problematical but ambitious work in progress - what it is in fact.

\section{AUTEUR}

\section{HÉLÈNE SPENGLER}

Université Grenoble Alpes

UMR LITT\&ARTS

Hélène Spengler enseigne les Lettres-Philosophie en classes préparatoires scientifiques au lycée Vaucanson de Grenoble. Elle est l'auteur de plusieurs études sur la Vie de Napoléon de Stendhal ainsi que d'une thèse sur L'Imaginaire et l'écriture de l'énergie dans l'œuvre de Stendhal : « La Révolution entre dans la littérature ", soutenue en 2006 à l'Université Stendhal-Grenoble 3. Elle est membre depuis 2000 du groupe de travail « Manuscrits de Stendhal » à l'université Grenoble Alpes, en tant que chercheur associée à la composante Charnières de LITT\&ARTS, UMR 5316. 